List your meeting here:

Following is a list of microscopy related meetings and courses. The editors would greatly appreciate input to this list via the electronic submission form under "events/other microscopy events" at the MSA webpage: www.microscopy.org. We will gladly add hypertext links to the notice on the web and insert a listing of the meeting in the next issue. Please furnish the following information (any additional information provided will be edited as required and printed on a space available basis):

- Meeting/Course name

- Meeting/Course dates

- Meeting/Course topic or short description

- Sponsoring society/organization/university

- Contact person

- Telephone number

- Fax number

- Email address

- URL for website

\section{General Links}

European Molecular Biology Organization Course \& Workshops

www.embo.org/programmes/courses-workshops.html

Gordon Research Conferences

www.grc.org/meetings.aspx? year $=2010$

Keystone Symposia

www.keystonesymposia.org/

Lehigh Microscopy School Courses

www.lehigh.edu/microscopy

Marine Biological Laboratory (MBL), Woods Hole, MA, USA

www.mbl.edu/education/

Royal Microscopical Society Annual Courses www.rms.org.uk/events/RMS_Annual_Courses

\section{Meetings 2015}

\section{Association for General and Applied} Microbiology, 2015 Annual Conference

March 1-4, 2015

Marburg, Germany

E-Mail: vaam-kongress@conventus.de

www.vaam-kongress2015.de

SCUR 2104: 42nd Annual Meeting of the Society for Cutaneous Ultrastructure Research

March 12-14, 2015

Mannheim, Germany

www.scur.org/content/e1319/SCUR_Flyer MANNHEIM_12_14th_March_2015.pdf
Society for Virology $25^{\text {th }}$ Annual

Meeting

March 18-21, 2015

Bochum, Germany

E-Mail: virology@conventus.de

www.virology-meeting.de

\section{Proteomics Forum 2015}

March 22-25, 2015

Berlin, Germany

www.proteomic-forum.de

FOM 2015: Focus on Microscopy 2015, $2^{\text {th }}$ International Conference

March 29-April 1, 2015

Göttingen, Germany

Email: info2015@FocusOnMicroscopy.org

www.focusonmicroscopy.org

$19^{\text {th }}$ International Conference on

Microscopy of Semiconducting

Materials (MSM-XIX)

March 29-April 2, 2015

Cambridge, UK

http://msm2015.iopconfs.org/home

\section{EBSD 2015 (Electron Backscatter}

Diffraction)

March 30-31, 2015

Sponsor: Royal Microscopical Society Glasgow, UK

www.rms.org.uk/events/Forthcoming_Events/ EBSD2015

2015 MRS Spring Meeting \& Exhibition April 6-10, 2015

San Francisco, CA

Sponsor: Materials Research Society

www.mrs.org/spring2015

\section{North Carolina Society of}

Histopathology Spring Meeting

April 9-11, 2015

Durham, North Carolina

Email: dwilliams@thehamner.org

NTM 2015: Novel Techniques in

\section{Microscopy}

April 12-15, 2015

Vancouver, Canada

http://osa.org/en-us/meetings/optics_and_ photonics_congresses/optics_in_the _ life_sciences/novel_techniques_in_ microscopy

\section{PICO 2015-Third Conference on}

\section{Frontiers of Aberration Corrected}

\section{Electron Microscopy}

April 19-23, 2015

Kasteel Vaalsbroek, The Netherlands www.er-c.org/pico2015/about.htm
The Biology of Regenerative

Medicines

April 22-24, 2015

Cambridge, UK

https://registration.hinxton.wellcome.ac.uk/ display_info.asp?id=432

$3^{\text {rd }}$ Croatian Microscopy Congress

April 26-29, 2015

Zadar, Croatia

www.irb.hr/users/avidos/1.\%20Newsletter_

Mikroskopijska.png

EMAS 2015: $14^{\text {th }}$ European Workshop on Modern Developments and

Applications in Microbeam Analysis

May 3-7, 2015

Portoroz, Slovenia

www.microbeamanalysis.eu/events/ event/6-emas-2015-14th-europeanworkshop-o

37th Annual Conference: International Cement Microscopy Association

(ICMA)

May 3-7, 2015

Seattle, WA

http://cemmicro.org

Tri-State Histology Meeting

May 6-8, 2015

Madison, Wisconsin

Email: judith.stasko@ars.usda.gov

http://msfh.wildapricot.org

ISM2015: $4^{\text {th }}$ Annual Meeting of the Israel Society for Microscopy

May 17-18, 2015

Ramat-Gan, Israel

www.ismicroscopy.org.il/ism2015

\section{$2^{\text {nd }}$ Workshop on EELS in Materials}

\section{Science}

May 18-20, 2015

Uppsala, Sweden

Sponsor: European Microscopy Society (EMS)

www.eurmicsoc.org/spons_events.html

$15^{\text {th }}$ International European Light

Microscopy Initiative (ELMI)

May 18-22, 2015

Barcelona, Spain

www.elmi2015.eu

\section{Botanical Microscopy 2015}

May 20-23, 2015

Exeter, UK

www.rms.org.uk/events/Forthcoming_Events/ BotanicalMicroscopy 


\section{Evactron ${ }^{\circledR}$ De-Contaminators}

\section{Fastest Removal of Performance Degrading Hydrocarbons}

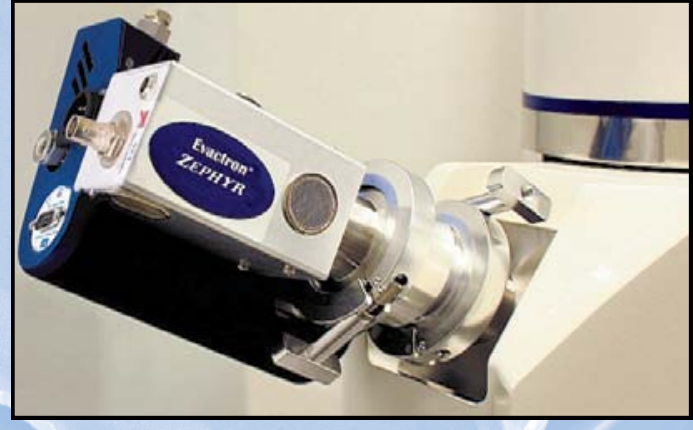

Evactron ${ }^{\circledR}$ Zephyr $^{\mathrm{TM}}$ remote plasma source

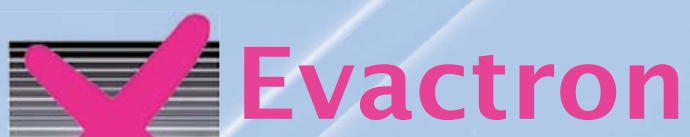

By XEI Scientific
- New Zephyr cleaning rate is $>100 \AA / \mathrm{min} @ 5$ mTorr $(0.6 \mathrm{~Pa}) 20 \mathrm{~cm}$ from plasma source on 20 liter chamber.

- Easy operation with turbo molecular pumps

- Interlock protects E-gun and detectors

- Proven safe for EDS windows -warranted.

- 2000 units sold, 5 year warranty

- Fully compliant: CE, SEMI S2, and NRTL standards

- Front panel control or remote computer commands
Refurbishing or servicing an electron microscope? Scope us out for your ion pump needs.

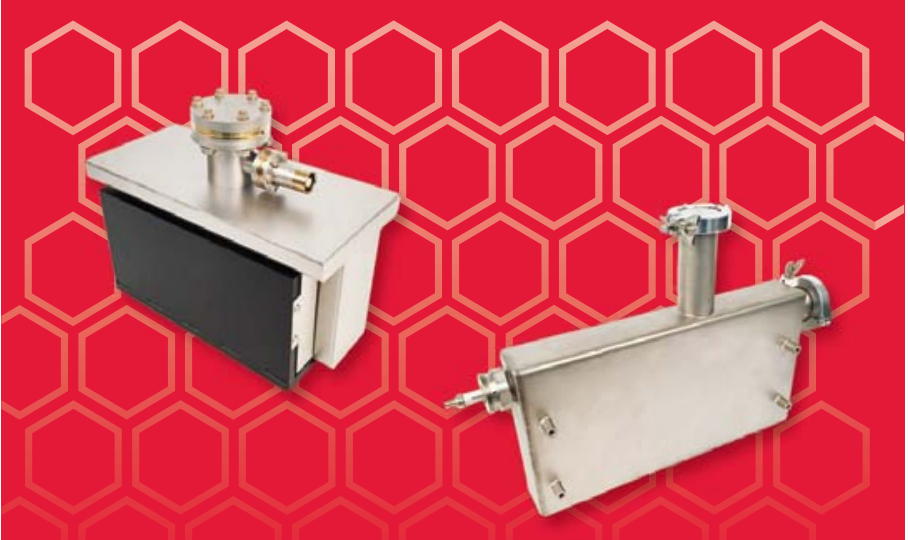

New | Rebuilt | Rebuilding Services DUTOKROM CORP. 800.446 .8811 WW
Reduce Your FIB Tool Operating Costs

with High Quality Replacement Parts from Oregon Physics

Regular replacement of aperture strip, extractor and suppressor parts is critical for maintaining the best performance in your FIB tool.

Oregon Physics makes it easy. Our parts meet exacting quality standards, while providing significant cost savings to our customers. And our fast turnaround helps assure your continued productivity.

Made to order aperture strips are our speciality. Provide your configuration and Oregon Physics will custom manufacture to your requirements.

Contact us to learn more about how Oregon Physics can help you reduce your FIB tool operating costs while improving tool productivity.

DREGON PHYSICS
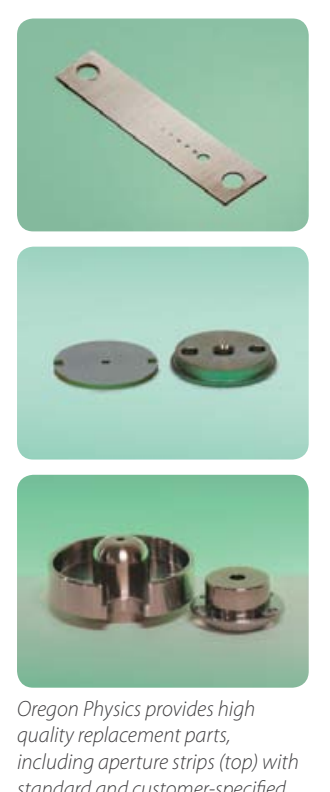

1.503.601.0041 oplab@oregon-physics.com www.oregon-physics.com 


\section{Southeastern Microscopy Society} 2015 meeting

May 20-22, 2015

Decatur, GA

http://southeasternmicroscopy. org/2015-meeting

\section{MSC-SMC Annual Meeting}

May 26-29, 2015

Hamilton, Ontario, Canada

Sponsor: Canadian Microscopy Society

www.bimr.ca/events/

msc-smc-annual-meeting-2015

\section{Inter/Micro 2015}

June 8-12, 2015

Chicago, IL

Sponsor: McCrone Research Institute

Phone: 312-842-7100

Email: intermicro@mcri.org

www.mcri.org

\section{SCANDEM2015}

June 9-11, 2015

Jyväskylä, Finland

www.jyu.fi/en/congress/scandem2015

$13^{\text {th }}$ Symposium on Bacterial Genetics and Ecology (BAGECO)

June 14-18, 2015

Milan, Italy

E-mail: bageco@conventus.de

www.bageco2015.org

\section{9th $^{\text {th }}$ American Chemical Society \\ Colloid and Surface Science \\ Symposium \\ June 15-17, 2015 \\ Pittsburgh, PA \\ Email: sg2e@andrew.cmu.edu \\ http://colloidssurfaces.org/upcoming-colloid- and-surface-science-symposia}

15 ${ }^{\text {th }}$ EMABM: $15^{\text {th }}$ Euroseminar on

Microscopy Applied to Building

Materials

June 16-19, 2015

Delft, The Netherlands

www.citg.tudelft.nl/emabm

$5^{\text {th }}$ International Colloids Conference

June 21-24, 2015

Amsterdam, The Netherlands

www.colloidsconference.com

Gordon Research Conference - Three

Dimensional Electron Microscopy

2015

June 21-26, 2015

New London

www.grc.org/programs.aspx?id=10864

8th Imaging the Cell 2015

June 24-26, 2015

Bordeaux, France

http://imagingthecell2015.com/index.php $57^{\text {th }}$ Electronic Materials Conference

(EMC)

June 24-26, 2015

Columbus, $\mathrm{OH}$

www.mrs.org/57th-emc-topics

$13^{\text {th }}$ European Meeting on

Ferroelectricity

June 28-July 3, 2015

Porto, Portugal

www.emf2015.org

MMC 2015: Microscience Microscopy

\section{Congress}

June 29-July 2, 2015

Manchester, UK

Sponsor: Royal Microscopical Society

www.mmc2014.org.uk/about/about-rms/mmc2015

$14^{\text {th }}$ ICSIA $-14^{\text {th }}$ International Congress

for Stereology \& Image Analysis

July 6-10, 2015

Liege, Belgium

www.14icsia.com

IAM Nano 2015: International Workshop on Advanced and In-situ Microscopies

of Functional Nanomaterials and

Devices

July 8-10, 2015

Hamburg, Germany

www.iamnano2015.com

$2^{\text {rd }}$ Annual International Conference on

Composites/Nano Engineering (ICCE-23)

July 12-18, 2015

Chengdu, China

www.icce-nano.org

Semicon West, 2015

July 14-16, 2015

San Francisco, CA

www.semiconwest2015.org

Quantitative Analysis of Grain Size July 16, 2015

York, UK

www.rms.org.uk/events/Forthcoming_Events/ GrainSize

Microscopy \& Microanalysis 2015

August 2-6, 2015

Portland, OR

Email: nicoleguy@conferencemanagers.com

www.microscopy.org

$64^{\text {th }}$ Annual Conference on Applications

of X-ray Analysis

August 3-7, 2015

Westminster, CO

www.dxcicdd.com

XXIV International Materials Research

Congress

August 16-20, 2015

Cancun, Mexico

www.mrs.org/imrc-2015
MCM2015: Multinational Congress on

Microscopy

August 23-29, 2015

Eger, Hungary

www.mcm2015.com

National Society for Histotechnology; 41 ${ }^{\text {st }}$ Annual Symposium/

Convention

August 28-September 2, 2015

Washington, DC

Sponsor: National Society for Histotechnology

Email: histo@nsh.org

www.nsh.org

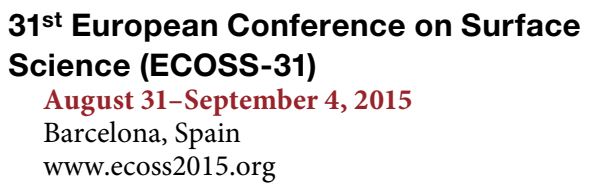

YUCOMAT 2015

August 31-September 4, 2015

Herceg Novi, Montenegro

www.mrs-serbia.org.rs/firstannouncement15. html

$33^{\text {rd }}$ Israel Vacuum Society Conference

(IVS)

September 6-8, 2015

Herzlia, Israel

Email: sidney.cohen@weizmann.ac.il

www.ivs.org.il/Meetings-and-workshops

\section{2015: Microscopy Conference} 2015

September 6-11, 2015

Göttingen, Germany

E-Mail: mc@conventus.de

www.mc2015.de

Microscopy at the Frontiers of Science 2015

September 9-11, 2015

Porto University, Portugal

$20^{\text {th }}$ International Conference on

Secondary Ion Mass Spectrometry

(SIMS XX)

September 13-18, 2015

Seattle, WA

Email: castner@uw.edu

www.simsxx.org

SMMS 2015 - Single-Molecule

Microscopy and Spectroscopy

September 14-16, 2015

London, UK

www.rsc.org/conferencesandevents/ rscconferences/fd/molecule-fd2015/index. asp

Intermetallics 2015

September 28-October 2, 2015

Bad Staffelstein, Germany

www.dgm-intermetallics.de 


\section{$3^{\text {rd }}$ International Meeting on \\ Nonbacterial Osteitis (CNO) \\ September 29-30, 2015 \\ Dresden, Germany \\ E-Mail: cno@conventus.de \\ www.issaid2015-congress.de/satellite- \\ symposium}

$8^{\text {th }}$ International Congress of Familial Mediterranean Fever and Systemic

Autoinflammatory Diseases

September 30-October 3, 2015

Dresden, Germany

E-Mail: issaid@conventus.de

www.issaid2015-congress.de

\section{$31^{\text {st }}$ North American Molecular Beam \\ Conference (NAMBE 2015) \\ October 4-7, 2015 \\ Mayan Riviera, Mexico \\ www2.avs.org/conferences/nambe/2015/index. htm}

\section{Materials Science \& Technology \\ 2015}

October 4-8, 2015

Columbus $\mathrm{OH}$

http://matscitech.org

Neuroscience 2015

October 17-21, 2015

Chicago, IL

Sponsor: Society for Neuroscience

www.sfn.org

\section{Shanghai Thin Films Conference \\ October 23-25, 2015 \\ Shanghai, China \\ Email: parsons@ncsu.edu,yongfeng.mei@ gmail.com \\ www.avs-shanghai.org}

\section{Single-Molecule Microscopy and \\ Spectroscopy \\ November 14-16, 2015 \\ London, UK \\ www.rsc.org/ConferencesAndEvents/ RSCConferences/FD/FD184/index.asp}

\section{MRS Fall Meeting \& Exhibition}

November 29-December 4, 2015

Boston, MA

Sponsor: Materials Research Society (MRS)

www.mrs.org/fall2015

\section{American Society for Cell Biology \\ (ASCB) 2015 Annual Meeting}

December 12-16, 2015

San Diego, CA

http://ascb.org/future-ascb-annual-meetings

Pacifichem 2015: The International Chemical Congress of Pacific Basin Societies

December 15-20, 2015

Honolulu, HI

www.pacifichem.org

\section{Courses 2015}

Raman Microspectroscopy

March 4-6, 2015

Westmont, IL

Hooke College of Applied Sciences

Phone: 630-887-7100

www.hookecollege.com

Hair Comparison

March 9-13, 2015

Westmont, IL

Hooke College of Applied Sciences

Phone: 630-887-7100

www.hookecollege.com

Modern Polarized Light Microscopy

March 16-20, 2015

Westmont, IL

Hooke College of Applied Sciences

Phone: 630-887-7100

www.hookecollege.com

Electron Microscopy Spring School

March 22-27, 2015

Leeds, UK

Sponsor: Royal Microscopical Society

www.rms.org.uk/events/Forthcoming_Events/ EMcourse

\section{Scanning Electron Microscopy}

March 23-27, 2015

Westmont, IL

Hooke College of Applied Sciences

Phone: 630-887-7100

www.hookecollege.com

\section{Modern Pollen Identification}

April 7-9, 2015

Chicago, IL

Sponsor: McCrone Research Institute

Phone: $312-842-7100$

Email: courses@mcri.org

www.mcri.org

\section{Applied Polarized Light Microscopy}

(PLM)/Forensic Microscopy

April 13-17, 2015

Sponsor: McCrone Research Institute

Phone: 312-842-7100

Email: courses@mcri.org

www.mcri.org

Analytical and Quantitative Light

Microscopy

April 29-May 8, 2015

Woods Hole, MA

www.mbl.edu/education/

special-topics-courses/

analytical-quantitative-light-microscopy

CIF Summer School: Electron

Diffraction Methods for Materials

Analysis

May 19-21, 2015

Fort Collins, CO

http://cif.colostate.edu/cif-summer-school $7^{\text {th }}$ Aberration-Corrected Electron

Microscopy and EELS School

June 1-5, 2015

Hamilton, Canada

McMaster University

Sponsor: Canadian Centre for Electron Microscopy

http://ccem.mcmaster.ca/outreach-courses.shtml

Neurobiology

June 6-August 2, 2015

Woods Hole, MA

www.mbl.edu/education/summer-courses/ neurobiology

MRI Bio-Cryo Course for Cellular

EM: EM Cryotechniques \& Cellular

Tomography

June 6-14, 2015

Salt Lake City, UT

Sponsors: Marinereef International \& University of Utah

Email: pdegeorge@marinereef.com

http://Marinereef.com/courses.php

Lehigh Microscopy School

June 7-12, 2015

Bethlehem, PA

Sponsor: Lehigh University

Email: slc6@lehigh.edu

www.lehigh.edu/microscopy

\section{Advanced Course on Cryo-Electron}

Tomography

June 8-12, 2015 (pre-course June 6-7)

Vienna, Austria

www.nexperion.net/expertise/cryotomo2015

\section{EMBO Practical Course: Advanced}

Electron Microscopy for Cell Biology

June 8-19, 2015

Bordeaux, France

Email: melina.petrel@u-bordeaux.fr

www.BIC.u-bordeaux2.fr

Physiology: Modern Cell Biology

Using Microscopic, Biochemical and

Computational approaches

June 14-August 2, 2015

Woods Hole, MA

www.mbl.edu/physiology

\section{Biology of Parasitism: Modern}

Approaches

June 19-August 8, 2015

Woods Hole, MA

http://courses.mbl.edu/BoP/Home.html

\section{Advanced Course Correlative Light}

Electron Microscopy

June 25-30, 2015

Utrecht, The Netherlands

www.cellbiology-utrecht.nl/em-courses/advancedcourse-correlative-light-electron-microscopy. html

\section{Microbial Diversity}

July 4-August 20, 2015

Woods Hole, MA

www.mbl.edu/microbialdiversity 
Getting the Most From Your Confocal

July 16-17, 2015

York, UK

Sponsor: Royal Microscopical Society

www.rms.org.uk/events/Forthcoming_Events/

GettingthemostfromyourConfocal

Frontiers in Host-Microbe Interactions August 3-20, 2015

Woods Hole, MA

www.mbl.edu/education/special-topics-courses/

frontiers-in-host-microbe-interactions

Zebrafish Development and Genetics

August 9-23, 2015

Woods Hole, MA

www.mbl.edu/zebrafish

\section{Electron Crystallography School - ECS2015 \\ August 28-31, 2015 \\ Duga Uvala, Croatia \\ www.blogs.uni-mainz.de/ecs2015}

\section{Optical Microscopy \& Imaging in the \\ Biomedical Sciences \\ September 9-19, 2015 \\ July 24-28, 2016 \\ Columbus, $\mathrm{OH}$ \\ Email: nicoleguy@conferencemanagers.com www.microscopy.org}

Woods Hole, MA

www.mbl.edu/education/special-topics-courses/ optical-microscopy-imaging-in-the-

biomedical-sciences/

Computational Image Analysis in

Cellular and Developmental Biology

October 11-21, 2015

Woods Hole, MA

www.mbl.edu/education/special-topics-courses/ computational-image-analysis

\section{Company Events 2015}

\section{EELS \& EFTEM Analysis Training School} 2015

April 14-17, 2015

Pleasanton, CA

Sponsor: Gatan

www.gatan.com/company/events/

eels-eftem-analysis-training-school-april-2015

\section{Meetings 2016}

Physics and Chemistry of Semiconductor

Surfaces and Interfaces (PCSI-43)

January 17-21, 2016

Location: TBD

Email: brillson.1@osu.edu

www.pcsiconference.org

\section{$24^{\text {th }}$ Australian Conference on}

Microscopy and Microanalysis

January 31-February 4, 2016

Melbourne, Australia

www.acmm2016.org

\section{MRS Spring Meeting \& Exhibition}

March 28-April 1, 2016

Phoenix, AZ

www.mrs.org/spring2016

\author{
American Society for Investigative \\ Pathology Annual Meeting \\ April 2-6, 2016 \\ San Diego, CA \\ www.asip.org/meetings/EB_future.cfm
}

ICMCTF'16 - International Conference

On Metallurgical Coatings \& Thin Films April 25-29, 2016

San Diego, CA

Email: pgreene@mrl.uiuc.edu

19 ${ }^{\text {th }}$ International Congress of Cytology

May 28-June 1, 2016

Pacifico Yokohama, Japan

www.cytologyjapan2016.com

\section{Ultrapath XVIII}

July 10-16, 2016

Lisbon, Portugal

www.ultrapath.org

Microscopy \& Microanalysis 2016

EMC 2016: 16 ${ }^{\text {th }}$ European Microscopy

\section{Congress}

August 28-September 2, 2016

Lyon, France

Sponsor: European Microscopy Society

http://emc2016.fr

National Society for Histotechnology; $42^{\text {nd }}$ Annual Symposium/

\section{Convention}

September 16-21, 2016

Long Beach, CA

Sponsor: National Society for Histotechnology

Email: histo@nsh.org

www.nsh.org

Neuroscience 2016

November 12-16, 2016

San Diego, CA

Sponsor: Society for Neuroscience

www.sfn.org

\section{American Society for Cell Biology}

(ASCB) 2016 Annual Meeting

December 3-7, 2016

San Francisco, CA

http://ascb.org/future-ascb-annual-meetings

\section{Meetings 2017}

American Society for Investigative

Pathology Annual Meeting

April 22-26, 2017

San Francisco, CA

www.asip.org/meetings/EB_future.cfm

Microscopy \& Microanalysis 2017

July 23-27, 2017

St. Louis, MO

Email: nicoleguy@conferencemanagers.com

www.microscopy.org
National Society for Histotechnology 43 ${ }^{\text {rd }}$ Annual Symposium/Convention September 15-20, 2017

Orlando, FL

Sponsor: National Society for Histotechnology Email: histo@nsh.org

www.nsh.org

\section{Neuroscience 2017}

November 11-15, 2017

Washington, DC

Sponsor: Society for Neuroscience

www.sfn.org

\section{American Society for Cell Biology \\ (ASCB) 2017 Annual Meeting \\ December 2-6, 2017 \\ Philadelphia, PA \\ http://ascb.org/future-ascb-annual-meetings}

\section{Meetings 2018}

American Society for Investigative

Pathology Annual Meeting

April 18-22, 2018

San Diego, CA

www.asip.org/meetings/EB_future.cfm

Microscopy \& Microanalysis 2018

August 5-9, 2018

Baltimore, MD

Email: nicoleguy@conferencemanagers.com www.microscopy.org

Neuroscience 2018

November 3-7, 2018

San Diego, CA

Sponsor: Society for Neuroscience

www.sfn.org

\section{American Society for Cell Biology}

(ASCB) 2018 Annual Meeting

December 8-12, 2018

San Diego, CA

http://ascb.org/future-ascb-annual-meetings

\section{Meetings 2019}

Microscopy \& Microanalysis 2019

August 4-8, 2019

Portland, OR

Email: nicoleguy@conferencemanagers.com

www.microscopy.org

\section{Neuroscience 2019}

October 19-23, 2019

Chicago, IL

Sponsor: Society for Neuroscience

www.sfn.org

\section{Meetings 2020}

Neuroscience 2020

October 24-18, 2020

Washington, DC

Sponsor: Society for Neuroscience

www.sfn.org 